\title{
Relación entre niveles de funcionalidad familiar y percepción de la calidad de vida en madres cabeza de familia habitantes de barrios vulnerables de la ciudad de Pereira
}

\author{
Marilyn Gallego Betancur ; Erika Tatiana Granda Serna; \\ Isabela Márquez Montoya ${ }^{3}$; José Alonso Andrade Salazar ${ }^{4}$
}

RESUMEN Investigación cuantitativa de tipo empírico analítico de corte transversal, cuyo objetivo es analizar la relación entre niveles de funcionalidad familiar y percepción de la calidad de vida, en 117 madres cabeza de familia habitantes de barrios vulnerables de la ciudad de Pereira, a las cuales se les aplicarán 3 instrumentos psicométricos para medir su funcionalidad familiar y su calidad de vida. Se encontró que 4 de cada 10 familias cuentan con algún tipo de disfunción familiar y que ello no afecta la percepción de una calidad de vida positiva, de modo que la relación es relativamente baja entre ambas variables.

Calidad de Vida;

PALABRAS

Disfunción Familiar;

Familia;

CLAVE

Funcionalidad Familiar,

Madre Cabeza de Hogar.

1 Estudiante de 8vo semestre de Psicología de la Fundación Universitaria del Área Andina seccional Pereira.mgallego23@estudiantes.areandina.edu.co

2 Estudiante de 8vo semestre de Psicología de la Fundación Universitaria del Área Andina seccional Pereira. egranda2@estudiantes.areandina.edu.co

3 Estudiante de 8vo semestre de Psicología de la Fundación Universitaria del Área Andina seccional Pereira. imarquez@estudiantes.areandina.edu.co

4 Psicólogo. Docente investigador Fundación Universitaria del Área Andina. Docente investigador Universidad de San Buenaventura Medellín extensión Armenia. Magister en Investigación Integrativa. PhD en Pensamiento complejo. Email: jandrade20@areandina.edu.co; jose.andrade@usbmed.edu.co 


\title{
Relationship between levels of family functionality and perception of the quality of life of mothers who are heads of households in vulnerable neighbourhoods of the city of Pereira
}

\begin{abstract}
This is a quantitative cross-sectional empirical research, whose objective is to analyze the relationship between levels of family functionality and perception of the quality of life in 117 mothers head of families living in vulnerable neighborhoods of the city of Pereira, to which 3 psychometric instruments will be applied to measure their family functionality and their quality of life. It was found that 4 out of 10 families have some type of family dysfunction and that this does not affect the perception of a positive quality of life, so that the relationship is relatively low between both variables.
\end{abstract}

Quality of Life;

Family Dysfunction;

KEYWORDS Family;

Family Functionality;

Mother Head of House. 


\section{INTRODUCCION}

La presente investigación tiene como meta, conocer la relación entre los niveles de funcionalidad familiar y percepción, que las madres cabeza de hogar tienen de la calidad de vida, de acuerdo a las tipologías de los hogares que cuentan con una mujer como cabeza de familia. De acuerdo a datos del Censo Nacional de Calidad de Vida, (DANE, 2010), se determinó que el 32,7\% de los hogares tiene la jefatura de una mujer, aspecto al que se suma un elevado grado de vulnerabilidad psicosocial, que implica su aumento, especialmente en madres y familias bajo diversas condiciones de vulnerabilidad (Castellanos, Garzón, \& Sotelo, 2015). En este tenor, se ha encontrado que "entre los hogares de bajo ingreso, los que están encabezados por mujeres enfrentan por lo general ciertos problemas, ya que ellas sufren discriminación en los mercados laborales o en los intentos de lograr apoyo para actividades generadoras de empleo o para la mejoría del hogar" (Hábitat, 1996, p. 6)

Cabe resaltar, que la calidad de vida se constituye en el estado de satisfacción global, derivado de la realización de las múltiples potencialidades de las personas (Zimmermann, 2006). Dicho esto, la calidad de vida cuenta con elementos subjetivos y objetivos, de allí que se determine como la sensación subjetiva de bienestar biopsicosocial, lo cual contiene aspectos como la intimidad, la enunciación emocional, el estado de seguridad y estabilidad percibida, así como también, el rendimiento personal y la salud en términos generales. No obstante, integra a la vez, elementos objetivos relacionados con el grado de bienestar material, el tipo de relaciones con el entorno, y la salud distinguida objetivamente (Ardila, 2003). La funcionalidad familiar es el elemento resultante de las interacciones familiares e integra elementos conflictivos y nutritivos (Satir, 1991, 2002)

La funcionalidad familiar o la salud familiar hace referencia al funcionamiento de la familia como sistema y agente social primario, razón por lo cual su adecuado o inadecuado funcionamiento resulta dinámico e inter-influyente en la preservación de la salud o también, en la emergencia de la enfermedad (Bronfenbrenner, 1987; Ruíz, Hernández, Mayrén, \& Vargas, 2014). Actualmente la conformación de las familias ha cambiado drásticamente, pasamos de tener una sociedad con familias mayormente nucleares con padre, madre e hijos, a evidenciar el crecimiento de las familias monoparentales con solo uno de sus padres presente, donde en mayor medida son las mujeres de su hogar, el único sustento de este.

Al ser la madre el único sustento de su familia, se pueden evidenciar deterioro en su calidad de vida y funcionalidad familiar, ya que no recibe apoyo económico ni moral de otro compañero/a. En el contexto patriarcal, además, la carga desproporcionada del cuidado de los hijos para las mujeres, no se compensa con un reconocimiento social de su labor. Por el contrario, suele considerarse poco valioso, en comparación con las labores tradicionales de provisión económica que realizan los hombres. Habitualmente el trabajo de las mujeres en la casa y su labor de maternidad se encuentran desvalorizados, dado que, al parecer "quedan fuera de la esfera del 
intercambio monetario y no se les puede medir en esos términos. Lo anterior refleja que los valores dentro de esa sociedad han reforzado la ideología de la inferioridad y la relativa carencia de poder que las mujeres poseen frente a los hombres" (Panatt \& Pardo, 2007, p. 42). En este sentido, es posible considerar la existencia de una relación entre percepción de calidad de vida y funcionalidad familiar, las mismas que podrían verse afectadas por el tipo de interacciones, tanto al interior de la familia como en sus relaciones e intercambios exteriores (Minuchin, 2003; Satir, 2002).

\section{MÉTODO}

Diseño: investigación cuantitativa, empírico-analítica de corte transversal.

Población: la investigación se desarrolló con ciento diecisiete (117) madres cabeza de familia, habitantes de barrios vulnerables de la ciudad de Pereira, realizada con un muestreo no probabilístico intencional.

Criterios de inclusión: mujeres madres cabeza de familia, habitantes de barrios vulnerables de la ciudad de Pereira.

Criterios de exclusión: hombres, no habitantes de barrios vulnerables, que residen fuera de la ciudad de Pereira, además de ser madre cabeza de familia.

Instrumentos: se aplicó una ficha de caracterización sociodemográfica que incluye los siguientes ítems: edad, género, escolaridad, religión, estrato, tipo de familia, número de hijos y tipo de trabajo. APGAR familiar de Smilkstein (Alegre \& Suárez, 2006). Los puntajes discriminan la presencia de Buena función familiar (18 - 20), Disfunción familiar leve $(14-17)$, Disfunción familiar moderada $(10-13)$ y Disfunción familiar severa (9 o menos). SF-36 de calidad de vida SF-36 de Ware y Sherbourne (1992).

Procedimiento: el procedimiento se llevó a cabo de la siguiente manera: Diseño/ aprobación proyecto de investigación; Aprobación bioética (Consentimiento informado); Firma del C.I, aplicación instrumentos; Análisis/sistematización datos resultados mediante el SPSS versión 18.0: La correlación de las variables se hizo mediante el coeficiente de correlación de Pearson. La fiabilidad de los instrumentos se hizo mediante el Alpha de Cronbach; elaboración de la discusión y del informe final; Devolución resultados.

\section{RESULTADOS}

De acuerdo a los resultados derivados de los instrumentos, la edad media de las madres encuestadas es de 36 a 46 años y en promedio tienen 2 hijos cada una; se encontró que las madres cabeza de familia presentan una elevada propensión $(71,8 \%)$ 
a llevar una vida en estado de soltería, mientras el $8,5 \%$ son separadas, $6,8 \%$ viudas, $6,0 \%$ son divorciadas y el $3,4 \%$ conviven en unión libre. Igualmente, se pone en evidencia que sus niveles de escolaridad son bajos, ya que el 39,3\% de las madres alcanzó un nivel de bachiller, 38,5\% estudió la primaria, y en ocasiones no completo, el $7,7 \%$ tiene un tecnólogo, $10,3 \%$ indican tener un técnico, y solo un $4,3 \%$ de estas alcanzaron un nivel universitario.

En cuanto al tipo de familia, el $80,3 \%$ de las familias son monoparentales, 10 cual indica que las madres son las únicas cuidadoras del hogar y aportantes económicamente en sus hogares, el 9,4\% de familias son extensas, 5,1\% extensa compuesta y el 5,1\% nuclear. Las madres en su mayoría $(82,1 \%)$ indican trabajar informalmente, generalmente como aseadoras o vendedoras ambulantes; el 17,9\% trabajan formalmente; de las cuales el 77,8\% no reciben ningún subsidio familiar ni ayuda externa.

\section{Tabla 1.}

Escolaridad

\begin{tabular}{llcccc}
\hline & & Frecuencia & Porcentaje & $\begin{array}{c}\text { Porcentaje } \\
\text { válido }\end{array}$ & $\begin{array}{c}\text { Porcentaje } \\
\text { acumulado }\end{array}$ \\
\hline Válido & Primaria & 45 & 38,5 & 38,5 & 38,5 \\
& Bachiller & 46 & 39,3 & 39,3 & 77,8 \\
& Técnico & 12 & 10,3 & 10,3 & 88,0 \\
& Tecnólogo & 9 & 7,7 & 7,7 & 95,7 \\
& Universitario & 5 & 4,3 & 4,3 & 100,0 \\
\hline & Total & 117 & 100,0 & 100,0 & \\
\hline
\end{tabular}

Fuente elaboración propia.

Tabla 2.

Estado Civil

\begin{tabular}{llcccc}
\hline & Frecuencia & Porcentaje & $\begin{array}{c}\text { Porcentaje } \\
\text { válido }\end{array}$ & $\begin{array}{c}\text { Porcentaje } \\
\text { acumulado }\end{array}$ \\
\hline Válido & Soltera & 84 & 71,8 & 71,8 & 71,8 \\
& Casada & 4 & 3,4 & 3,4 & 75,2 \\
& Divorciada & 7 & 6,0 & 6,0 & 81,2 \\
& Unión Libre & 4 & 3,4 & 3,4 & 84,6 \\
& Viuda & 8 & 6,8 & 6,8 & 91,5 \\
& Separada & 10 & 8,5 & 8,5 & 100,0 \\
\hline & & & & & \\
\hline
\end{tabular}

Fuente elaboración propia. 
Tabla 3 .

Tipos de familia.

\begin{tabular}{llcccc}
\hline & & Frecuencia & Porcentaje & $\begin{array}{c}\text { Porcentaje } \\
\text { válido }\end{array}$ & $\begin{array}{c}\text { Porcentaje } \\
\text { acumulado }\end{array}$ \\
\hline Válido & nuclear & 6 & 5,1 & 5,1 & 5,1 \\
& extensa & 11 & 9,4 & 9,4 & 14,5 \\
& extensa compuesta & 6 & 5,1 & 5,1 & 19,7 \\
& monoparental & 94 & 80,3 & 80,3 & 100,0 \\
\hline & Total & 117 & 100,0 & 100,0 & \\
\hline
\end{tabular}

Fuente elaboración propia.

De acuerdo a los resultados derivados del SF-36, que se encarga de evaluar la calidad de vida, se encontró: el estado de salud física de las madres es preocupantemente deteriorado en general: $88,9 \%$ mala, destacando que el dolor corporal que se presenta en una parte específica del cuerpo, puede llegar a limitar la ejecución de su trabajo o la realización de sus actividades cotidianas; el 44,4 \% de las madres presenta un dolor moderado que ralentiza y dificulta la realización de sus actividades cotidianas, más no las impide; pese a esto, el 73,5\% de las madres afirman no tener limitaciones, y el $65 \%$ aseguran haberse sentido múltiples veces durante el último mes, llenas de vitalidad frente al sentimiento de cansancio.

En cuanto a la función social, se evidenció que al 78,6\% de las madres les afecta poco su salud física y emocional con relación a su ámbito social, que se refleja al mismo tiempo en el poco porcentaje $(28,2 \%)$ de madres que redujeron el tiempo dedicado al trabajo o a sus actividades cotidianas a causa de algún problema emocional, en divergencia a todos los datos encontrados; finalmente se demuestra que aunque no dejaron de hacer sus actividades por ninguna razón, el 59\% de ellas llegó a sentirse deprimida, ansiosa o nerviosa.

Como dato general, se pudo inferir que las percepciones que tienen las madres acerca de su salud es excelente en el 91,5\%, puesto que ellas al ser el sustento de su familia no pueden verse limitadas por ningún factor.

\section{Tabla 4.}

Sumatoria SF36

\begin{tabular}{llcccc}
\hline & Frecuencia & Porcentaje & $\begin{array}{c}\text { Porcentaje } \\
\text { válido }\end{array}$ & $\begin{array}{c}\text { Porcentaje } \\
\text { acumulado }\end{array}$ \\
\hline Válido & Excelente & 107 & 91,5 & 91,5 & 91,5 \\
& Muy buena & 10 & 8,5 & 8,5 & 100,0 \\
\hline & Total & 117 & 100,0 & 100,0 & \\
\hline
\end{tabular}

Fuente elaboración propia. 
Según los resultados del APGAR familiar (mide la funcionalidad familiar), se encontró que un $50,4 \%$ de las madres tiene una mala capacidad para utilizar recursos tanto dentro de la familia como por fuera de ella para adaptarse al cambio y resolver problemas en situaciones de crisis, en consecuencia, el 59\% de ellas percibe la participación que su familia le brinda como mala; por otro lado, el apoyo que las madres han recibido por parte de su familia para emprender ha sido en un 59,8\% mala. Como reflejo de estos aspectos las madres reciben poco afecto por parte de sus familiares, el 53\% lo perciben como malo y no las comprenden cuando experimentan estados de alegría, tristeza, angustia, etc.

De manera general las madres perciben en un $59,8 \%$ que tienen una buena funcionalidad familiar.

Tabla 5 .

Tipos de funcionalidad familiar.

SUMA FF

\begin{tabular}{llcccc}
\hline & & Frecuencia & Porcentaje & $\begin{array}{c}\text { Porcentaje } \\
\text { válido }\end{array}$ & $\begin{array}{c}\text { Porcentaje } \\
\text { acumulado }\end{array}$ \\
\hline Válido & BFF & 70 & 59,8 & 59,8 & 59,8 \\
& FFL & 25 & 21,4 & 21,4 & 81,2 \\
& FFM & 10 & 8,5 & 8,5 & 89,7 \\
& FFG & 12 & 10,3 & 10,3 & 100,0 \\
\hline & Total & 117 & 100,0 & 100,0 & \\
\hline
\end{tabular}

Fuente: elaboración propia

\section{DISCUSIÓN}

Según el objetivo de esta investigación, la cual fue encontrar los niveles de funcionalidad familiar y su relación con la calidad de vida, se halló que existe una disfuncionalidad presente, manifiesta en el tipo de interacciones intrafamiliares lejanas, distantes, de poca comprensión y apoyo- y que, a pesar de ello, la percepción de calidad de vida es por lo general buena, aun cuando 4 de cada 10 familias presenta algún tipo de disfuncionalidad. En este tenor conviene señalar que, las madres cabeza de hogar $(\mathrm{MCH})$ constituyen una de las poblaciones vulnerables que requiere ayuda y asistencia en salud mental, física y en apoyo económico y laboral (Andrade \& Valencia, 2011), aspecto relacionado al hecho de que las madres evaluadas no solo se encuentran en riesgo social, sino también los miembros de la familia que dependen de ellas. 
En las MCH prevalecen problemas como la inseguridad laboral, el incremento de la pobreza, un nivel educativo bajo, violencia de género entre otras, que deterioran su capacidad de qué hacer frente al estrés y también de abandonar los territorios de conflicto y depresión social (Bravo, 2005; Russell \& Harmes, 2006), no obstante, cada vez más mujeres de múltiples regiones, etnias, condiciones sociales y estratos socio-económicos asumen el rol de proveedoras primordiales de sus hogares y son registradas por otros como cabeza de hogar y líderes de sus proyectos familiares (Organización Panamerica de la Salud - OPS, 2009; Roca \& Riccardi, 2014). Estos elementos permiten pensar en una elevada resiliencia, la cual puede ser irradiada a través de conductas de protección, cuidado, comprensión y apego positivo con sus semejantes (Andrade \& Sicachá, 2012; Suárez, 1996). En ellas la calidad de vida es más que el equivalente a la suma de los puntajes de las condiciones de vida objetivamente medibles en una persona, tales como salud física, condiciones de vida, estado ambiental del entorno y percepción medio ambiental de la familia, relaciones sociales, actividades funcionales u ocupación (Zimmermann, 2006) y en realidad constituye una apuesta vital realizada de forma natural ante lo adverso de su situación biopsicosocial (Cyrulnik, 2003; Puig \& Rubio, 2013).

En las MCH la calidad de vida implica vivenciar un estado de satisfacción general, procedente de la realización de las potencialidades como persona y como familia, aspecto que se ha encontrado en investigaciones similares sobre el tema, y que no se aleja de la experiencia de una funcionalidad familiar relativamente estable (Andrade \& Buitriago, 2011; Andrade \& Valencia, 2011; Bronfenbrenner, 1987; Castellanos, Garzón, \& Sotelo, 2015; Roca \& Riccardi, 2014). Cabe mencionar que en ellas la funcionalidad familiar y la calidad de vida comportan aspectos subjetivos relacionadas con la interpretación de las relaciones y objetivos que giran en torno a las vivencias y metas alcanzadas en cuanto a la convivencia y la estabilidad socioeconómica; de allí que aspectos como la falta de apoyo cuando se establecen nuevos proyectos, y la concentración en temas como el trabajo con una escasa participación social, puedan estar relacionados con la disfuncionalidad percibida, aspecto resaltado como importante al momento de comprender la funcionalidad o disfuncionalidad familiar (Minuchin, 2003; Satir, 1991).

\section{CONCLUSIONES}

Acorde al análisis de datos, se evidencia una propensión a familias de tipo monoparental, donde la madre cabeza de hogar de sectores vulnerables, constituye el único sustento de su hogar.

Se observa que las mujeres entre los 17 a 25 años presentan una mala participación dentro de su entorno familiar. La funcionalidad familiar en las madres cabeza de hogar es significativamente mala, y en ella se puede evidenciar que las familias no cumplen y/o ejercen un papel de acompañamiento, por lo cual no se perciben entre los miembros buenas relaciones, ya que, por lo general, un elevado porcentaje de las 
madres cabeza de hogar no se sienten satisfechas con el apoyo que les brindan sus familias y la comprensión que reciben de estas en el aspecto emocional.

Conviene mencionar que, a pesar de la disfuncionalidad familiar y de los bajos niveles económicos a los que se encuentran sometidas, se descubrió que su percepción sobre su calidad de vida es buena, ya que encuentran un nivel de confort apropiado en su hogar; en este tenor, señalan que su salud física es "muy bueno", puesto que la mayoría de ellas están muy activas diariamente, por lo que no dejan de hacer sus diligencias cotidianas ya sea de tipo familiar, laboral, recreativa o social. Lo anterior indica que las madres cabeza de familia en su día a día, tienen que hacer un esfuerzo constante para poder llevar a cabo sus actividades cotidianas, pero lo interpretan de forma positiva, lo cual puede hacer referencia a un elevado nivel de resiliencia, especialmente porque de ello depende en gran medida, el sustento de su familia.

1. Alegre, Y., \& Suárez, M. (2006). Instrumentos de Atención a la Familia: El Familiograma y el APGAR familiar (fascículos Cadec). Rampa, 1(1), 48-57. Retrieved from http://www.idefiperu.org/RAMNRO1/N9C-PG48-CADEC Instrum Familia2A.pdf

2. Andrade, J.A., \& Buitriago, G. (2011). Estado de la función familiar, en población desplazada privada de la libertad en la cárcel "Peñas blancas" de Calarcá Quindío en el último semestre de 2009. Revista Aletheia De La Corporación Universitaria Empresarial Alexander Con Humboldt, 1, 1-13. Retrieved from https:/es.scribd.com/document/75496822/Estado-de-la-funcion-familiar-endesplazados-privados-de-la-libertad-by-Jose-Alonso-Andrade-salazar

3. Andrade, J. A., \& Sicachá, M. (2012). Indicadores de salud mental comunitaria en madres cabeza de hogar desplazadas. El Ágora Usb, 12(1), 61-76. Retrieved from http://www.scielo.org.co/scielo.php?script=sci_arttext\&pid $=$ S1657-80312012000100004

4. Andrade, J. A., \& Valencia, L. M. (2011). Descripción de la función familiar de veinte mujeres víctimas de violencia intrafamiliar psicológica, de la comuna cuatro del municipio de armenia en el 2010. Revista Aletheia De La Corporación Universitaria Empresarial Alexander Con Humbold, 1(1), 53-72. Retrieved from http://cue.edu.co/wp-content/uploads/2015/05/REVISTA_DE_ INVESTIGACIN_ALETHEYA.pdf

5. Ardila, R. (2003). Calidad de vida: una definición integradora. Revista Latinoamericana de Psicología, 35(2), 161-164.

6. Bravo, R. (2005). Las metas del milenio y la igualdad de género: el caso de la República. (Comisión Económica para América Latina y el Caribe - CEPAL, Ed.). Santiago de Chile: United Nations Publications. 
7. Bronfenbrenner, U. (1987). La Ecología del Desarrollo Humano. Buenos Aires: Paidós.

8. Castellanos, Y., Garzón, M., \& Sotelo, C. (2015). Factores que influyen en las madres cabeza de familia para acceder a los procesos de formación que proporciona el banco caja social a sus colaboradores. Bogotá: Universidad Piloto de Colombia.

9. Cyrulnik, B. (2003). Los patitos feos, la resiliencia una infancia infeliz no determina la vida (5ta ed.). Barcelona: Gedisa.

10. Minuchin, S. (2003). Familias y terapia familiar. Barcelona: Editorial Gedisa.

11. Organización Panamerica de la Salud - OPS. (2009). Manual de familias fuertes. Washington, D.C: Organización Panamericana de la Salud, Programa Mujer, Salud y Desarrollo. Retrieved from http://www1.paho.org/hq/ dmdocuments/2010/Manual Familias Fuertes Guia para el Facilitador.pdf

12. Panatt, M., \& Pardo, M. (2007). La mujer trabajadora jefa de hogar y su articulación doméstico laboral. Bogotá: Universidad Academia de Humanismo Cristiano.

13. Puig, G., \& Rubio, J. (2013). Manual de resiliencia aplicada. Barcelona: Editorial GEDISA.

14. Roca, M., \& Riccardi, A. (2014). Familia: Funcionalidad y efectividad como red de apoyo social en pacientes con depresión que están ingresados en el área de hospitalización de psiquiatría del hospital del IESS Dr. Teodoro Maldonado Carbo en la ciudad de Guayaquil (Spanish). Informes Psicológicos, 14(2), 81102.

15. Ruíz, O., Hernández, M., Mayrén, J., \& Vargas, M. (2014). Family Functioning of Consumers of Addictive Sustances With and Without Criminal Behavior. Liberabit, 20(1), 109-119.

16. Russell, D., \& Harmes, R. (2006). Feminicidio: una perspectiva global. New York: Teacher College Press.

17. Satir, V. (1991). Family Therapy and Beyond. New York: Science and Behavior Books.

18. Satir, V. (2002). Nuevas relaciones humanas en el núcleo familiar. México D.F: Editorial Pax México.

19. Suárez, E. (1996). El concepto de resiliencia comunitaria desde la perspectiva de la promoción de salud. In M. Kotliarenco, C. Álvarez, \& I. Cáceres (Eds.), Resiliencia: Construyendo en adversidad (pp. 51-64). Santiago de Chile: CEANIM.

20. Zimmermann, M. (2006). Psicologia ambiental, calidad de vida y desarrollo sostenible. Barcelona: Editorial Ecoe. 


\section{PREPNDINP | MEEMRO DE LARED}

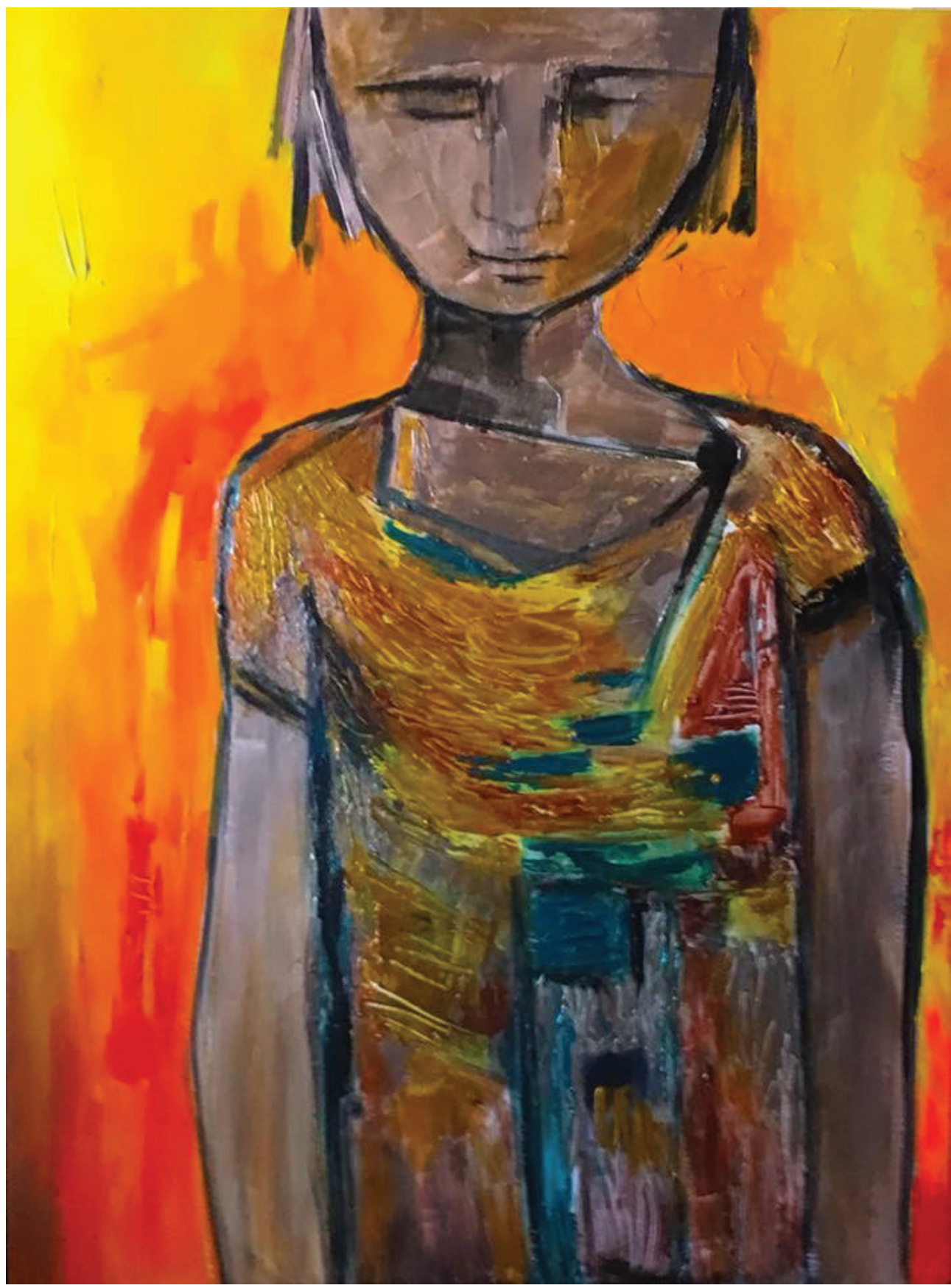

Acrílico sobre lienzo. "Niña a-dorada", Virginia Aristizábal Parra. 